\title{
Teste de provocação oral aberto na confirmação de alergia ao leite de vaca mediada por IgE: qual seu valor na prática clínica?
}

\author{
Open oral food challenge in the confirmation of cow's milk allergy mediated by lgE: what is its value in \\ the clinical practice?
}

Raquel Bicudo Mendonça ${ }^{1}$, Renata Rodrigues Cocco $^{1}$, Roseli Oselka S. Sarni' ${ }^{2}$ Dirceu Solé3

\section{RESUMO}

Objetivo: Revisar os principais protocolos de padronização para o teste de provocação oral aberto aplicado a crianças com suspeita de alergia ao leite de vaca mediada por imunoglobulina E.

Fontes de dados: Foram selecionados artigos publicados, nos últimos dez anos, nas bases de dados Medline, Lilacs e SciELO, utilizando-se os descritores de assunto: "hipersensibilidade alimentar", "leite de vaca", "alergia ao leite de vaca”, "teste de provocação oral”, “crianças” e "diagnóstico”.

Síntese dos dados: O teste de provocação oral é o método mais fidedigno para estabelecer ou excluir o diagnóstico de alergia alimentar e sua forma aberta pode ser a primeira opção, quando apenas reações objetivas são esperadas. O local para realizar o teste deve possuir todos os recursos para tratamento de emergência. Como preparo, o paciente deve seguir dieta de exclusão do alimento suspeito e descontinuar o uso de medicamentos que possam interferir no resultado do teste. Habitualmente, utilizam-se 8 a $10 \mathrm{~g}$ de leite em pó ou $100 \mathrm{~mL}$ de leite fluido, oferecidos em volumes progressivos, a intervalos de dez a 60 minutos. Apesar da rigidez do método, ainda existem situações em que há dificuldade de interpretação dos resultados; por isso, médicos bem treinados devem aplicar o teste, sendo cautelosos ao julgarem as reações.

Conclusões: Mesmo considerando as limitações que dificultam a aplicação do teste de provocação oral na prática clínica, a implementação do teste nos serviços de saúde poderia reduzir diagnósticos falsos-positivos de alergias.

Palavras-chave: hipersensibilidade alimentar; substitutos do leite humano; diagnóstico.

\section{ABSTRACT}

Objective: To review the main standardization protocols of the open oral food challenge applied to children with suspicion of cow's milk allergy mediated by immunoglobulin E.

Data source: Medline, Lilacs and SciELO databases were searched for articles published in the last ten years using the following descriptors: "food hypersensitivity", "cow's milk", "cow's milk allergy”, "oral food challenge”, "children” and "diagnosis".

Data synthesis: The oral food challenge test is the most reliable method to establish or exclude the diagnosis of food allergy and the open challenge can be the first option when only objective reactions are expected. All medications and equipment needed for emergency treatment should be available. Before being submitted to the test, the patient must follow a diet excluding the suspected food and discontinue the use of medications that may interfere with the results of the test. Usually, it is performed using 8-10g of powdered milk or $100 \mathrm{~mL}$ of fluid milk, offered in progressive volumes
Instituição: Disciplina de Alergia e Imunologia Clínica do Departamento de Pediatria da Escola Paulista de Medicina da Universidade Federal de São Paulo (EPM/Unifesp), São Paulo, SP, Brasil

${ }^{1}$ Mestre em Ciências pelo Departamento de Pediatria da EPM/Unifesp, São Paulo, SP, Brasil

Doutora em Ciências pelo Departamento de Pediatria da EPM/Unifesp, São Paulo, SP, Brasil

${ }^{3}$ Livre-docente da Unifesp; Professor Titular da Disciplina de Alergia e Imunologia Clínica do Departamento de Pediatria da EPM/Unifesp, São Paulo, SP, Brasil
Endereço para correspondência:

Raquel Bicudo Mendonça

Rua dos Otonis, 725 - Vila Clementino

CEP: 04025-002 - São Paulo/SP

E-mail: raquelbicudo.nutri@hotmail.com

Conflito de interesse: nada a declarar

Recebido em: 7/9/2010

Aprovado em: 20/1/2011 
at intervals of ten to 60 minutes. Despite the rigour of the method, there are still situations difficult to interpret. Therefore, well-trained doctors must administer the test, being cautious about their clinical judgment.

Conclusions: Although there are some restrictions in the application of the oral food challenge test with cow's milk in the clinical practice, its implementation in health services could reduce false positive diagnoses of allergies.

Key-words: food hypersensitivity; breast-milk substitutes; diagnosis.

\section{Introdução}

Alergia alimentar (AA) é a denominação utilizada para caracterizar reações adversas a alimentos que envolvem mecanismo imunológico ${ }^{(1)}$, sendo divididas em: mediadas por imunoglobulinas $\mathrm{E}(\mathrm{IgE})$, não mediadas por $\operatorname{IgE} \mathrm{e}$ mistas $^{(2)}$.

As reações mediadas por $\mathrm{IgE}$ ocorrem em minutos até duas horas após a exposição à(s) proteína(s) alergênica(s). Entre elas podem ser listadas reações cutâneas (urticária, prurido, angioedema, hiperemia e eritema perioral), reações gastrintestinais (edema e prurido de lábios, língua e palato, náuseas, vômitos, cólica e diarreia imediatos), reações respiratórias (coriza, prurido nasal, espirros, hiperemia e prurido ocular, broncoespasmo agudo, tosse e edema de laringe) e reações sistêmicas (anafilaxia com hipotensão e choque) ${ }^{(2-4)}$.

A alergia ao leite de vaca (ALV) é a AA mais frequente em lactentes, tendo prevalência de $2-3 \%$ em crianças com idade inferior a um ano em países desenvolvidos ${ }^{(5,6)}$. Habitualmente, a ALV é transitória e a maioria das crianças adquire tolerância por volta dos três anos de idade ${ }^{(7,8)}$.

O método utilizado para estabelecer ou excluir o diagnóstico de AA ou para determinar a aquisição de tolerância ao alimento é o teste de provocação oral (TPO), que consiste na oferta do alimento ao paciente, em doses progressivas, sob supervisão médica, realizado após um período de dieta de exclusão do alimento suspeito ${ }^{(9,10)}$.

$\mathrm{Na}$ ALV mediada por $\operatorname{IgE}$, a sensibilização às proteínas lácteas pode ser verificada pela deteç̧ão de IgE sérica específica (teste in vitro) ou pelo teste cutâneo de hipersensibilidade imediata $(\mathrm{TCHI})^{(11)}$. Tais testes apresentam valor preditivo positivo e negativo de 50 e $95 \%$, respectivamente ${ }^{(12,13)}$ Portanto, servem apenas para verificar a sensibilização ao alérgeno, não sendo suficientes para estabelecer o diagnóstico de AA se a reatividade clínica não for observada ${ }^{(14)}$.

Diversos estudos foram publicados na tentativa de estabelecer pontos de corte para os testes in vivo (TCHI) e in vitro, com valor preditivo elevado (90 a 95\%) de ocorrer uma reação positiva por ocasião da ingestão do alimento, buscando excluir a necessidade de realização do teste de provocação oral. No entanto, os valores estabelecidos variam consideravelmente entre os alimentos testados e populações estudadas e, desta forma, não levam a indicações ou contraindicações absolutas quanto à realização do $\mathrm{TPO}^{(15-18)}$.

O tratamento da ALV consiste na eliminação de leite de vaca (LV) e derivados da dieta, além de produtos e preparações contendo esses alimentos ${ }^{(8)}$. Crianças submetidas à dieta isenta de LV e derivados sem orientação adequada estão sob risco nutricional e podem apresentar repercussões no crescimento ponderoestatural e no desenvolvimento ${ }^{(19-22)}$. Além disso, a dieta de exclusão é relativamente mais onerosa, pode trazer prejuízos psicossociais à criança, ser inconveniente à família e de difícil monitoração fora do ambiente domiciliar ${ }^{(23)}$. Portanto, é muito importante que o diagnóstico da ALV seja feito de maneira criteriosa, evitando expor a criança e sua família à restrição alimentar desnecessária ${ }^{(24)}$.

Os benefícios de um TPO positivo incluem a confirmação do diagnóstico de AA, a redução do risco de exposição acidental, a diminuição da ansiedade sobre o desconhecido e a validação do esforço do paciente e seus familiares em evitar o alimento. Os benefícios de um TPO negativo são a liberação de ingestão do alimento suspeito, com consequente redução do risco nutricional e melhora na qualidade de vida do paciente ${ }^{(9)}$.

O objetivo deste artigo foi revisar a literatura e salientar os tópicos mais importantes relacionados ao TPO aberto aplicado a crianças com suspeita de ALV mediada por IgE, com base em protocolos de padronização do TPO. Para tal, foram selecionados artigos publicados nos últimos dez anos nas bases de dados Medline, Lilacs e SciELO. As palavras-chave utilizadas como fonte de busca foram: "hipersensibilidade alimentar", "leite de vaca", "alergia ao leite de vaca", "teste de provocação oral”, "crianças” e "diagnóstico”.

\section{Tipos de teste de provocação oral}

Há três tipos de TPO: a) aberto, quando o alimento é oferecido em sua forma natural, com o conhecimento do paciente, familiares e do médico; b) simples cego, quando o alimento é mascarado, de forma que o paciente não reconheça se está ingerindo o alimento suspeito ou placebo, e apenas o 
médico conhece o que está sendo administrado; c) duplo cego controlado por placebo (DCCP), no qual o alimento testado e placebo são preparados e codificados por uma terceira pessoa não envolvida na avaliação do paciente, reduzindo a influência de ambos, paciente e observador ${ }^{(9,25)}$. O método duplo cego é o mais fidedigno dos testes empregados no diagnóstico de AA. Por esse motivo, é o teste mais indicado para protocolos científicos e quando apenas sintomas subjetivos são esperados $^{(16)}$. Porém, na prática clínica diária, sua utilização é limitada pelos custos e recursos humanos envolvidos e pelo tempo necessário para sua realização ${ }^{(4)}$.

Entende-se por sintomas subjetivos aqueles que podem ser influenciados por fatores psicológicos, como aversão ao alimento ou ansiedade. Como exemplos podem ser citados: dor ou desconforto abdominal, náuseas, vômito, mal-estar geral, palpitação, exacerbação de prurido cutâneo (no caso de dermatite atópica), prurido labial, prurido em orofaringe, queimação na língua, sensação de aperto na garganta, dificuldade em engolir e outros sintomas, como sonolência e irritabilidade ${ }^{(10,26,27)}$.

O TPO simples cego pode ser feito com ou sem placebo, na dependência do protocolo adotado ${ }^{(9)}$. De qualquer forma, os critérios para o mascaramento são os mesmos adotados para o duplo cego controlado por placebo, ou seja, a preparação deve mascarar o sabor, o odor, a aparência e a consistência do alimento testado e, se houver placebo, este deve ser indistinguível do alimento testado. A elaboração de tais preparações é um desafio para os nutricionistas ${ }^{(28,29)}$. Como o TPO simples cego possui as mesmas dificuldades do duplo cego controlado por placebo, o último é preferível por afastar a influência por parte do observador ${ }^{(16)}$.

Após um TPO simples cego ou duplo cego controlado por placebo negativo, deve-se realizar uma fase aberta do teste com a oferta do alimento em quantidade equivalente a uma porção habitualmente consumida de acordo com a idade do paciente ${ }^{(16,30)}$. Apesar de o TPO duplo cego controlado por placebo ser mais rigoroso que o aberto, um artigo de revisão recente afirma que as indicações para realização do teste duplo cego controlado por placebo seriam restritas a pesquisas científicas e situações específicas na prática clínica, por exemplo, após TPO aberto com manifestações clínicas subjetivas isoladas (prurido oral ou náuseas, por exemplo) ${ }^{(9)}$.

O TPO aberto é eficiente, relativamente barato e particularmente importante, porque apenas cerca de um terço das suspeitas de AA têm o diagnóstico confirmado a partir de um TPO positivo. Tendo em vista a maior praticidade, comparativamente ao duplo cego controlado por placebo, considera-se que o TPO aberto pode ser a primeira opção quando a necessidade de realização do teste de provocação é estabelecida ${ }^{(9)}$.

\section{Teste de provocação oral aberto com leite de vaca}

Segundo Nowak-Wegrzyn et a ${ }^{(9)}$, o TPO aberto deve ser o teste de escolha em pacientes com grande chance de apresentar resultado negativo, como aqueles em dieta de exclusão do alimento suspeito, com relatos de ausência de sintomas às transgressões dietéticas, ou ainda em pessoas com suspeita clínica de ALV mediada por IgE sem confirmação laboratorial da presença de IgE específica. Em outras palavras, o TPO aberto é especialmente indicado nos casos em que se pretende afastar o diagnóstico de AA.

O TPO aberto deve ser realizado quando apenas sinais e sintomas clínicos objetivos são esperados ${ }^{(16,30,31)}$. Entende-se por sintomas objetivos aqueles facilmente observados e/ou graves, insistentes ou reprodutíveis ${ }^{(10)}$. É importante considerar que, enquanto um resultado claramente negativo afasta a hipótese de alergia alimentar, um resultado positivo apenas com sintomas subjetivos pode necessitar de confirmação posterior por provocação oral cega ${ }^{(32)}$.

Em crianças menores de um ano, raramente sintomas subjetivos estão envolvidos e, portanto, o TPO aberto pode ser usado com a mesma confiabilidade atribuída ao duplo cego controlado por placebo ${ }^{(32)}$. Segundo a Academia Européia de Alergia e Imunologia Clínica, o TPO aberto pode ser aplicado em crianças com até três anos de idade, pois nelas sintomas subjetivos são raramente observados ${ }^{(16)}$. O TPO aberto não é recomendado para crianças maiores de três anos ou adultos, quando há suspeita de que sintomas subjetivos possam ocorrer ${ }^{(16,32)}$.

As demais situações em que o TPO pode ser contraindicado (ou adiado) incluem aquelas nas quais o paciente possui história recente convincente de reação anafilática associada à presença de IgE específica, asma não controlada, uso de medicamentos que interfiram com o resultado do teste ou com o tratamento de possíveis reações e na vigência de condições clínicas que possam atrapalhar a interpretação do teste $\mathrm{e}^{(8,9,16)}$.

\section{Infraestrutura física e recursos humanos}

O local para a realização do TPO deve possuir todos os equipamentos para tratamento de emergência ${ }^{(16)}$. Em pacientes sem história de reações graves, não há necessidade de internação em unidade hospitalar. Nesses casos, o TPO pode ser realizado em ambulatório ou consultório, desde que 
o ambiente seja seguro (disponibilidade de materiais e equipamentos para atendimento de emergência), livre do contato com pacientes portadores de doenças infecciosas, limpo, confortável e apropriado para permanência do paciente por longo período. Médicos e enfermeiros bem treinados devem acompanhar o paciente durante todo o teste ${ }^{(11,32)}$.

\section{Aspectos éticos}

Os riscos e benefícios do teste devem ser informados de maneira clara aos responsáveis para posterior obtenção do consentimento por escrito ${ }^{(11)}$.

\section{Orientações pré-teste}

Antes do TPO, a criança com suspeita de ALV mediada por IgE deve passar por um período de uma a duas semanas de exclusão de proteínas do LV e derivados ${ }^{(9,12,24,30-32)}$. O paciente deve descontinuar o uso de medicamentos que possam interferir no resultado do teste. Como regra geral, os anti-histamínicos devem ser suspensos, mas não há consenso de quanto tempo antes do TPO o paciente deve evitar esse medicamento. Entre os trabalhos publicados, a recomendação varia de três a dez dias $^{(9,11,12,24,32-34)}$. Corticosteroides inalatórios podem ser mantidos em doses mínimas suficientes para controlar a asma, e os corticosteroides tópicos podem ser aplicados em pequenas áreas da pele ${ }^{(11,12,24)}$. Nessa fase, a orientação da família quanto à dieta de exclusão, inclusive leitura e interpretação de rotulagem de alimentos e bula de medicamentos, é essencial ${ }^{(35)}$.

Entre os estudos que relatam a indicação de jejum prévio à realização do teste, não há uniformidade quanto à duração, variando de duas a oito horas (Tabela 1). Crianças de baixa idade, que não conseguem fazer jejum prolongado, podem consumir metade da porção usual de uma refeição leve de acordo com a idade, duas horas antes do teste $e^{(9,12,24,30)}$. A principal finalidade do jejum é facilitar a absorção do alimento testado, evitar que a ingestão prévia de alimentos interfira na interpretação dos resultados e reduzir o risco ao paciente, caso sejam necessárias manobras de ressuscitação ${ }^{(9)}$.

Mesmo que sigam todas as orientações pré-teste, apenas devem ser submetidos ao TPO pacientes em boas condições clínicas e com suas doenças alérgicas (asma, rinite e dermatite atópica) controladas ou em fase de remissão ${ }^{(9,16)}$.

\section{Protocolos de testes}

\section{Alimento ofertado}

No TPO aberto, sugere-se oferecer o alimento na forma e quantidade habitualmente consumida, de acordo com a idade ${ }^{(16,32,36)}$. Outros alimentos, como derivados de LV, também podem ser utilizados para o teste (Tabela 1). Para o TPO com LV, habitualmente utiliza-se 8-10g de leite em pó ou $100 \mathrm{~mL}$ de leite fluido ${ }^{(11,12,24,30)}$.

Os alimentos usados em TPO aberto podem ser trazidos de casa pelo familiar do paciente, após orientação, ou preparados no local. O local envolvido na preparação deve ser limpo e livre de risco de contaminação com leite ou outros alimentos que possam causar reações no paciente ${ }^{(9)}$.

É importante lembrar que, em protocolos científicos, independentemente do alimento escolhido, deve-se padronizar a qualidade e a quantidade de proteína que está sendo oferecida para permitir a comparação dos resultados com o mesmo rigor metodológico ${ }^{(27)}$.

\section{Doses e intervalos}

Alguns estudos sugerem que a dose inicial seja menor do que a necessária para deflagrar sintomas na criança, de acordo com o referido pelos responsáveis ${ }^{(16,24,30,36)}$. Outros especificam que o volume inicial deve ser de 0,1 ou $1 \mathrm{~mL}^{(9,11,12,16)}$. As doses são aumentadas progressivamente, sendo dobradas ${ }^{(9,11,16,24,30,36)}$ ou seguindo uma escala logarítmica $\left(1,3,10,30,100 \mathrm{~mL}^{(9,16)}\right.$. O tempo de intervalo entre as doses varia de dez a 60 minutos, segundo os diversos trabalhos que trazem essa recomendação (Tabela 1).

\section{Período de observação após o teste}

O paciente deve permanecer sob observação médica após a administração da última dose de alimento. Esse período costuma variar de 1 a 2 horas, de acordo com os trabalhos publicados. Niggemann e Beyer ${ }^{(32)}$ sugerem que o período seja de 24 horas.

\section{Monitoramento de sinais e sintomas clínicos}

Durante a realização do teste, os sinais vitais precisam ser monitorados, e um exame físico minucioso deve ser realizado, verificando-se ruídos pulmonares e a pele da criança, antes de administrar nova dose de alimento ${ }^{(9)}$.

O TPO é interrompido imediatamente caso se observe algum sinal ou sintoma objetivo característico de AA mediada por $\operatorname{Ig} E^{(9)}$. Eritema apenas no local onde houver contato com o LV, como manifestação isolada, e presença de sinais e sintomas subjetivos isolados em crianças acima de 1ano não devem levar à interrupção do teste ${ }^{(37)}$.

\section{Tratamento das reações}

A criança deverá receber o tratamento de acordo com a gravidade e a natureza das reações ${ }^{(9)}$. No caso de reações cutâneas 


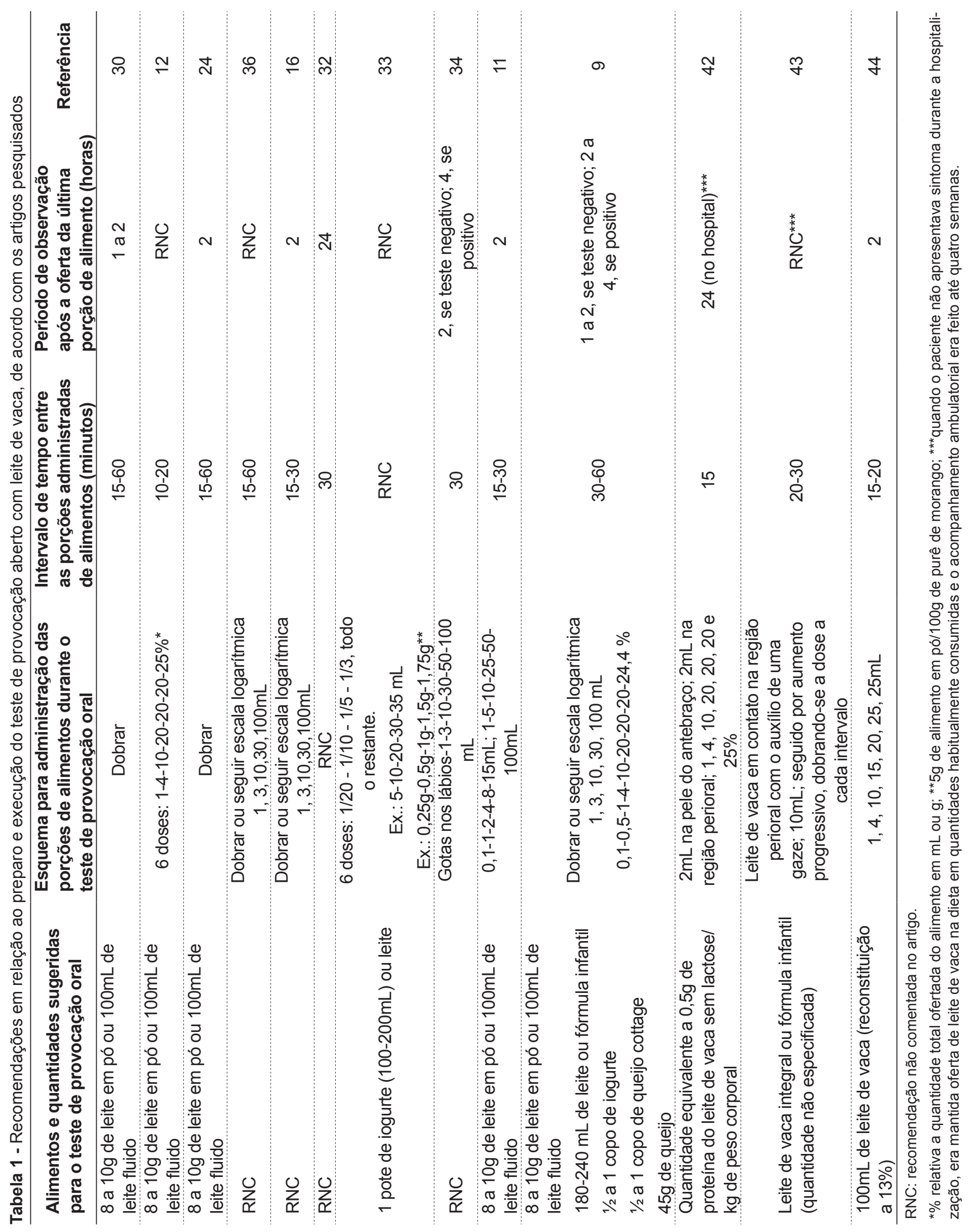


leves e moderadas, administrar doses de anti-histamínicos usuais para o peso e idade da criança, preferencialmente por via oral. Corticosteroides orais poderão ser prescritos para uso domiciliar, mas seu efeito terapêutico é mais tardio em relação aos anti-histamínicos. Sintomas gastrintestinais (vômitos e diarreia) precisam ser monitorados conforme sua intensidade; antieméticos e/ou medidas apropriadas para hidratação poderão ser administrados de acordo com a avaliação do médico responsável. A adrenalina intramuscular é aplicada imediatamente na região vastolateral da coxa em caso de comprometimento dos sistema respiratório (crise de broncoespasmo) ou cardiovascular (hipotensão).

Em relação à necessidade de acesso venoso durante a realização do TPO para facilitar o tratamento de possíveis reações, considera-se que tal precaução deva ser tomada apenas em crianças pequenas com asma e/ou história prévia de reação grave $e^{(3,16,32)}$.

\section{Interpretação dos resultados}

As manifestações clínicas consideradas características de ALV mediada por IgE são: urticária, pápulas, angioedema, hiperemia, prurido (cutâneo, labial, orofaríngeo, nasal ou ocular), coriza nasal, espirros, tosse, chiado, lacrimejamento, náuseas e vômitos ${ }^{(38,39)}$. Os testes são considerados positivos nos seguintes $\operatorname{casos}^{(37)}$ : quando as manifestações são intensas ou repetidas; quando mais de uma manifestação é observada, envolvendo um ou mais sistemas; quando os sinais e sintomas, mesmo que subjetivos, são observados em crianças menores de um ano de idade.

Apesar de o TPO ser considerado o método mais confiável para o diagnóstico de reações relacionadas a alimentos, especialmente quando o método duplo cego é empregado, ainda existem situações em que o resultado pode ser questionável ${ }^{(10,37,40)}$.

Eritemas observados apenas nos locais de contato com o LV podem representar apenas reações cutâneas, e o alimento pode ser bem tolerado se a administração oral prosseguir, resultando em TPO negativo. Por outro lado, placas de urticária disseminadas pelo corpo são sinais claros e objetivos relacionados à $\mathrm{AA}^{(10)}$. Resíduos de alimentos nos lábios, na face e nas mãos devem ser removidos com água para evitar eventual irritação de contato e confundir a interpretação dos resultados do $\mathrm{TPO}^{(9)}$.

Alguns sinais e sintomas, apesar de serem facilmente observados, podem representar uma reação de origem psicológica, devendo ser interpretados com cuidado. No caso de vômito, por exemplo, um único episódio é motivo para interromper o TPO. São necessários episódios repetidos para considerar o resultado do teste positivo ${ }^{(37)}$.

De qualquer forma, a interpretação dos resultados é questão bastante delicada em um TPO, não havendo na literatura definição exata de quais sintomas traduzem um resultado positivo e qual seria a gravidade de cada sinal. Sintomas como dor abdominal, náuseas, prurido labial e sensação de aperto na garganta relatados por pacientes, por exemplo, podem ser considerados subjetivos em alguns casos, enquanto, em outros, podem fazer parte do quadro de reação anafilática ${ }^{(3,40)}$. Por isso, Nowak-Wegrzyn et a ${ }^{(9)}$ referem que, em crianças pequenas, especialmente as que ainda não sabem falar, atitudes como colocar a mão na boca, coçar a língua, arranhar o pescoço ou mudança de comportamento podem ser indícios de uma reação grave e, nesses casos, dependendo do nível de desconforto do paciente e do julgamento do médico, o TPO deve ser interrompido e o tratamento adequado deve ser administrado.

\section{Comentários}

Embora o TPO seja reconhecido na literatura como um método de extrema importância por ser um procedimento demorado, trabalhoso e dispendioso, raramente é empregado por profissionais de saúde em nosso meio e ainda não faz parte do rol de procedimentos diagnósticos reconhecidos pelos sistemas de saúde público e privado. Assim, o diagnóstico de AA habitualmente é estabelecido com base na história clínica, exame físico, exames subsidiários que avaliam a presença de $\operatorname{IgE}$ específica e melhora dos sinais e sintomas a partir da instituição de uma dieta de exclusão do alimento suspeito.

Porém, em relação ao fato de o TPO ser considerado um método diagnóstico dispendioso, devido ao tempo e recursos humanos necessários para sua realização, deve-se contrapor os custos de uma dieta de exclusão. Afinal, a manutenção de uma criança em uso de fórmulas especiais para tratamento de ALV pode ser oneroso à família e/ou aos serviços de saúde. Vale lembrar ainda que dietas de exclusão realizadas sem acompanhamento de especialistas podem comprometer a qualidade de vida e o estado nutricional da criança.

A Secretaria do Estado da Saúde de São Paulo publicou em 2007 um protocolo de distribuição gratuita de fórmulas infantis especiais para crianças com ALV e idade inferior a dois anos ${ }^{(41)}$. O protocolo indica a realização do TPO para esclarecimento diagnóstico de alergia e verificação de tolerância 
ao LV. Porém, nota-se haver limitações da aplicação do teste na prática clínica, tendo em vista a falta de padronização do método, a dificuldade de execução e, por fim, a não remuneração do teste, uma vez que este não consta nas tabelas de procedimentos diagnósticos vigentes no país.

A implementação do TPO nos serviços de saúde possibilitaria o correto diagnóstico da ALV, reduzindo gastos e riscos desnecessários impostos pela dieta de exclusão de LV. Recentemente, foram publicados dois estudos brasileiros relatando os resultados de TPO abertos aplicados para a confirmação diagnóstica de $\mathrm{ALV}^{(42,43)}$. Um terceiro estudo $^{(44)}$, ainda não publicado, voltado especificamente para confirmação de ALV mediada por IgE, também foi realizado. Os protocolos utilizados em cada um dos estudos brasileiros estão descritos na Tabela 1 . Um deles foi realizado no Ambulatório de Gastroenterologia Pediátrica do Hospital das Clínicas da Universidade Federal de Pernambuco e envolveu 65 crianças com história de sintomas atribuídos à ingestão de $\mathrm{LV}$, das quais $53 \%$ apresentaram TPO positivo ${ }^{(42)}$. O outro foi realizado nos ambulatórios de Gastroenterologia Pediátrica da Universidade Federal de São Paulo (Unifesp) e do Hospital Pequeno Príncipe em Curitiba, envolvendo 121 pacientes em dieta de exclusão de $\mathrm{LV}$ e derivados, dos quais $23 \%$ apresentaram TPO positivo, todos com manifestações tardias ${ }^{(43)}$. O estudo ainda não publicado foi realizado nos ambulatórios de Alergia Pediátrica da Unifesp e da Universidade Federal de Sergipe com 46 crianças com história de reações imediatas à ingestão de LV, das quais $41 \%$ tiveram confirmação diagnóstica por meio do $\mathrm{TPO}^{(44)}$. Nota-se, nos três estudos, a alta porcentagem de crianças que tiveram a liberação de LV na dieta após a realização do TPO (47, 77 e 59\%, respectivamente). Tais resultados corroboram a necessidade do TPO para confirmar o diagnóstico de ALV ou para verificar a aquisição de tolerância oral ao LV.

Espera-se que a presente revisão estimule maior reflexão por parte dos profissionais de saúde quanto à importância da realização do TPO para confirmar ou excluir o diagnóstico de ALV. Além disso, espera-se que os serviços de saúde se empenhem em implementar protocolos seguros, reprodutíveis e padronizados para o TPO, de maneira a possibilitar novos e amplos estudos sobre alergia alimentar.

\section{Referências bibliográficas}

1. Johansson SG, Bieber T, Dahl R, Friedmann PS, Lanier BQ, Lockey RF et al. Revised nomenclature for allergy for global use: Report of The Nomenclature Review Committee of the World Allergy Organization, October 2003. J Allergy Clin Immunol 2004;113:832-6.

2. Cianferoni A, Spergel JM. Food allergy: review, classification and diagnosis. Allergol Int 2009;58:457-66 .

3. Muraro A, Roberts G, Clark A, Eigenmann PA, Halken S, Lack G et al. The management of anaphylaxis in childhood: position paper of the European academy of allergology and clinical immunology. Allergy 2007;62:857-71.

4. Sociedade Brasileira de Pediatria; Associação Brasileira de Alergia e Imunopatologia. Consenso Brasileiro sobre Alergia Alimentar: 2007. Rev Med Minas Gerais 2008;18 (Supl 1):S1-44.

5. Crittenden RG, Bennett LE. Cow's milk allergy: a complex disorder. J Am Coll Nutr 2005;24 (6 Suppl ):582S-91

6. Høst A. Frequency of cow's milk allergy in childhood. Ann Allergy Asthma Immunol 2002;89 (6 Suppl 1):33-7.

7. Skripak JM, Matsui EC, Mudd K, Wood RA. The natural history of IgE-mediated cow's milk allergy. J Allergy Clin Immunol 2007;120:1172-7.

8. Benhamou AH, Schiäppi Tempia MG, Belli DC, Eigenmann PA. An overview of cow's milk allergy in children. Swiss Med Wkly 2009;139:300-7.

9. Nowak-Wegrzyn A, Assa'ad AH, Bahna SL, Bock SA, Sicherer SH, Teuber SS; Adverse Reactions to Food Committee of American Academy of Allergy, Asthma \& Immunology. Work Group report: oral food challenge testing. J Allergy Clin Immunol 2009;123 (6 Suppl):S365-83.

10. Niggemann B. When is an oral food challenge positive? Allergy 2010;65:2-6.

11. Ito K, Urisu A. Diagnosis of food allergy based on oral food challenge test. Allergol Int 2009;58:467-74.

12. Sicherer SH. Food allergy: when and how to perform oral food challenges. Pediatr Allergy Immunol 1999;10:226-34.
13. Crespo JF, James JM, Rodriguez J. Diagnosis and therapy of food allergy Mol Nutr Food Res 2004;48:347-55.

14. Cochrane S, Beyer K, Clausen M, Wjst M, Hiller R, Nicoletti C et al. Factors influencing the incidence and prevalence of food allergy. Allergy 2009;64:124655.

15. American College of Allergy, Asthma, \& Immunology. Food allergy: a practice parameter. Ann Allergy Asthma Immunol 2006;96 (3 Suppl 2):S1-68.

16. Bindslev-Jensen C, Ballmer-Weber BK, Bengtsson U, Blanco C, Ebner C, Hourihane $\mathrm{J}$ et al. Standardization of food challenges in patients with immediate reactions to foods - position paper from the European Academy of Allergology and Clinical Immunology. Allergy 2004;59:690-7.

17. Niggemann B, Rolinck-Werninghaus C, Mehl A, Binder C, Ziegert M, Beyer $\mathrm{K}$. Controlled oral food challenges in children - when indicated, when superfluous? Allergy 2005;60:865-70.

18. Miceli Sopo S, Radzik D, Calvani M. The predictive value of specific immunoglobulin $E$ levels for the first diagnosis of cow's milk allergy. A critical analysis of pediatric literature. Pediatr Allergy Immunol 2007;18:575-82.

19. Black RE, Williams SM, Jones IE, Goulding A. Children who avoid drinking cow milk have low dietary calcium intakes and poor bone health. Am J Clin Nutr 2002;76:675-80.

20. Christie L, Hine RJ, Parker JG, Burks W. Food allergies in children affect nutrient intake and growth. J Am Diet Assoc 2002;102:1648-51.

21. Medeiros LC, Speridião PG, Sdepanian VL, Fagundes-Neto U, Morais MB. Nutrient intake and nutritional status of children following a diet free from cow's milk and cow's milk by-products. J Pediatr (Rio J) 2004;80:363-70.

22. Noimark L, Cox HE. Nutritional problems related to food allergy in childhood Pediatr Allergy Immunol 2008;19:188-95.

23. Sampson HA, Scanlon SM. Natural history of food hypersensitivity in children with atopic dermatitis. J Pediatr 1989;115:23-7. 
24. Sampson HA. Food allergy. Part 2: diagnosis and management. J Allergy Clin Immunol 1999;103:981-9.

25. Bernstein IL, Li JT, Bernstein DI, Hamilton R, Spector SL, Tan R et al. Allergy diagnostic testing: an updated practice parameter. Ann Allergy Asthma Immunol 2008;100 (3 Suppl 3):S1-148.

26. Taylor SL, Hefle SL, Bindslev-Jensen C, Atkins FM, Andre C, Bruijnzeel-Koomen $\mathrm{C}$ et al. A consensus protocol for the determination of the threshold doses for allergenic foods: how much is too much? Clin Exp Allergy 2004;34:689-95.

27. Vlieg-Boerstra BJ, van der Heide S, Bijleveld CM, Kukler J, Duiverman EJ, Dubois AE. Placebo reactions in double-blind, placebo-controlled food challenges in children. Allergy 2007;62:905-12.

28. Huijbers GB, Colen AA, Jansen JJ, Kardinaal AF, Vlieg-Boerstra BJ, Martens BP. Masking foods for food challenge: practical aspects of masking foods for a double-blind, placebo-controlled food challenge. J Am Diet Assoc 1994;94:645-9.

29. Vlieg-Boerstra BJ, Bijleveld CM, van der Heide S, Beusekamp BJ, WoltPlompen SA, Kukler J et al. Development and validation of challenge materials for double-blind, placebo-controlled food challenges in children. J Allergy Clin Immunol 2004;113:341-6.

30. Bock SA, Sampson HA, Atkins FM, Zeiger RS, Lehrer S, Sachs M et al. Doubleblind, placebo-controlled food challenge (DBPCFC) as an office procedure: a manual. J Allergy Clin Immunol 1988;82:986-97.

31. Venter C, Pereira B, Voigt K, Grundy J, Clayton CB, Gant C et al. Comparison of open and double-blind placebo-controlled food challenges in diagnosis of food hypersensitivity amongst children. J Hum Nutr Diet 2007;20:565-79.

32. Niggemann B, Beyer K. Diagnosis of food allergy in children: toward a standardization of food challenge. J Pediatr Gastroenterol Nutr 2007;45:399-404.

33. Mukoyama T, Nishima S, Arita M, Ito S, Urisu A, Ebisawa M et al. Guidelines for diagnosis and management of pediatric food allergy in Japan. Allergol Int 2007; 56:349-61.

34. Kneepkens CM, Meijer Y. Clinical practice. Diagnosis and treatment of cow's milk allergy. Eur J Pediatr 2009;168:891-6.

35. Mofidi S. Nutritional management of pediatric food hypersensitivity. Pediatrics 2003;111:1645-53.

36. Bindslev-Jensen C. Standardization of double-blind, placebo-controlled food challenges. Allergy 2001;56 (Suppl 67):75-7.

37. Niggemann B, Beyer K. Pitfalls in double-blind, placebo-controlled oral food challenges. Allergy 2007;62:729-32.

38. Perry TT, Matsui EC, Conover-Walker MK, Wood RA. Risk of oral food challenges. J Allergy Clin Immunol 2004;114:1164-8.

39. Sampson HA. Update on food allergy. J Allergy Clin Immunol 2004;113:805-19.

40. Gellerstedt M, Magnusson J, Gråjö U, Ahlstedt S, Bengtsson U. Interpretation of subjective symptoms in double-blind placebo-controlled food challenges interobserver reliability. Allergy 2004;59:354-6.

41. Secretaria do Estado da Saúde [homepage on the Internet]. Resolução SS - 336, de 27 de novembro de 2007 [cited 2010 Feb 4]. São Paulo: Diário Oficial do Estado de São Paulo; 2007. Available from: http://www.saude. sp.gov.br/resources/geral/acoes_da_sessp/assistencia_farmaceutica/ protocolo_leites.pdf

42. Lins MG, Horowitz MR, Silva GA, Motta ME. Oral food challenge test to confirm the diagnosis of cow's milk allergy. J Pediatr (Rio J) 2010;86:285-9.

43. Correa FF, Vieira MC, Yamamoto DR, Speridião PG, Morais MB. Teste de desencadeamento aberto no diagnóstico de alergia à proteína do leite de vaca. J Pediatr (Rio J) 2010;86:163.

44.Mendonça RB. Teste de provocação oral aberto na confirmação de alergia ao leite de vaca mediada por imunoglobulina $E$ [tese de mestrado]. São Paulo (SP): Unifesp; 2010. 